\title{
Translation of Gas Spectroscopy into the Clinic, a Promising Tool for Non-Invasive Diagnostics in Respiratory Health Care of Neonates
}

\author{
Andrea Pacheco ${ }^{1,2 *}$, Eugene Dempsey ${ }^{3}$ and Stefan Andersson-Engels ${ }^{1,2}$ \\ ${ }^{1}$ Department of Physics, University College Cork, Ireland \\ ${ }^{2}$ IPIC, Tyndall National Institute, Ireland \\ ${ }^{3}$ Cork University Maternity Hospital Wilton, Cork, Ireland
}

*Corresponding author: Andrea Pacheco, IPIC Tyndall National Institute, Lee Maltings Complex Dyke Parade, Cork, T12 R5CP, Ireland.

To Cite This Article: Andrea Pacheco* and Stefan Andersson-Engels. Translation of Gas Spectroscopy into the Clinic, a Promising Tool for NonInvasive Diagnostics in Respiratory Health Care of Neonates. 2020 - 9(3). AJBSR.MS.ID.001399. DOI: 10.34297/AJBSR.2020.09.001399.

Received: 制: June 24, 2020; Published: 阱 July 06, 2020

\begin{abstract}
Respiratory Distress Syndrome (RDS) is the leading cause of death among preterm infants worldwide. The latest improvements made in tackling RDS are focused on less invasive surfactant therapy and non-invasive ventilation. As a consequence of the more advanced and intensive respiratory support, there is an increase in the prevalence of infants with chronic lung disease. The current first line techniques used to monitor at risk infants with respiratory distress are pulse oximetry, chest radiography (X-ray) and blood gas analysis with X-ray imaging being the main diagnostic tool used. However, ionized radiation can be harmful for the infant and exposure should be kept to the minimum requirement.

Translation of the gas spectroscopy to the clinic could provide additional information about alveolar composition and make the step forward in objective medical assessment for RDS in neonates. Lack of direct, non-invasive method of measuring the oxygen concentration in the lung instead of oxygen saturation in blood, precludes understanding of underlying cause of the RDS and real time monitoring of the response of neonates under treatment. Gas spectroscopy has proven successful in quantifying gas content inside cavities surrounded by solids or liquids, and thus may be used to assess the oxygen content in the lungs of a neonate. This non-invasive light-based technology opens a new range of tools for lung function assessment, as it can be used to map continuously the oxygen presence and the changes in the volume of the lungs during respiration. Its superior specificity makes it a good candidate for the early diagnosis of respiratory distress. In addition, it could also be integrated to existing equipment to help optimize respiratory support during treatment.
\end{abstract}

Keywords: Respiratory distress; Neonatal healthcare; Non-invasive; Gas spectroscopy

Abbreviations: RDS: Respiratory Distress Syndrome; CLD: Chronic Lung Disease; MRI: Magnetic Resonance Imaging; GASMAS: Gas in Scattering Media Absorption Spectroscopy; IR: Infrared; CPAP: Continuous Positive Airway Pressure; CT: Computed Tomography

\section{Introduction}

The motivation for this article is that each year an estimated of 15 million infants are born preterm (before 37 completed weeks of gestation) [1]. $95 \%$ of neonates at 24 weeks gestational age and $80 \%$ of infants born at 28 weeks have respiratory distress syndrome (RDS) [2]. RDS in neonates is due to a deficiency of surfactant, which is a protein produced by the cell lining in the alveoli of the foetus, which reduces the surface tension of pulmonary fluids and contributes to the elasticity of the lungs. It is detectable from approximately week 23 onward and enables the platform for gas exchange [3]. The deficiency of surfactant results in reduced lung compliance increased respiratory effort and increased likelihood of alveolar collapse [4]. RDS-specific interventions, therapies and advanced care technologies including administration of antenatal corticosteroids, surfactant, alternative modes of non-invasive ventilation and ventilatory strategies such as high-frequency oscillatory ventilation have reduced the neonatal mortality rate over the past two decades [5]. However, mechanical ventilation can have a bad effect on a lung that is developing or in the process of repairing. The longer mechanical ventilation is administrated, the more likely is for the infant to develop chronic lung dysfunction (CLD). 
Although current research is aiming for less invasive and more targeted diagnostics and treatment techniques [6], the main tool is X-ray imaging such as chest radiograph or rarely volumetric computed tomography (CT) with the drawback of radiation burden [7]. Alternative non harmful diagnostic tools are magnetic resonance imaging (MRI) and ultrasound imaging. The image quality of MRI can be affected by heartbeat and respiration motion and there is still on-going work to fully achieve lung imaging comparable to CT [8]. Lung ultrasound imaging has been proven to be of good use for bedside differential diagnosis of RDS in new-borns $[9,10]$.

While the above-mentioned imaging techniques enable the localization of gas filled cavities in the body, none of them make it possible for the quantification of alveolar gas composition in real time. Gas in Scattering Media Absorption Spectroscopy (GASMAS) is a non-invasive optical technique which can be used to measure continuously oxygen concentration and inflated volume locally in the lung [11]. The clinical applications of this novel technology are currently being explored and this article provides insights into the potential improvements that could rise from translating GASMAS as a rapid non- radiographic bedside detection of alveolar oxygen gas for neonatal respiratory health care.

\section{Principles of GASMAS technique}

Optical spectroscopy is widely used to provide information on different gas species [12]. GASMAS refers to the regime where the absorbing gas is inside a highly scattering media and relies on the difference between the absorptive imprints between solid-state materials and free gas. The absorption spectrum of free oxygen inside the alveoli is 10,000 times narrower than those of blood, and other surrounding tissues of the thoracic cavity. The deepest penetration of light in human tissue corresponds to wavelengths between $650 \mathrm{~nm}$ and 1.4 microns. Since molecular oxygen has a wide range of absorption lines around $760 \mathrm{~nm}$ and water vapor has numerous lines around 820 and $980 \mathrm{~nm}$, the selection of light sources for clinical applications of GASMAS in respiratory health care are restricted to these wavelengths [13].

Previous feasibility studies demonstrated that gas sensing in the lung can be achieved by sending low-intensity laser light through the thoracic wall and detecting the emerging light with a photodetector, which reveals the absorption imprints of oxygen and water vapor [14]. The Beer-Lambert law is used to assess the gas concentration. In scattering media, the path length is not well defined; hence two wavelengths are necessary to tune across the absorption lines of water vapor and molecular oxygen simultaneously. By making the assumption that optical properties are the same for the two wavelengths, the absorption signal from oxygen is normalized by the water vapor one and then the absolute concentration of oxygen is calculated [15].

\section{Potential clinical application of GASMAS}

In the transition from intrauterine environment to air, immediately after birth, the neonate fills the airways down to the alveolar level with air to start breathing and gas exchange. Preterm neonates are born with lungs still in prenatal stages. The lack of surfactant results in increase respiratory effort to expand the lung which has weak alveoli due to RDS. By enabling the localization of regions in the lung with poorly inflated alveoli, GASMAS technology could be a key support for clinicians in the diagnostics and quantification of surfactant deficiency. The probes could be placed over the thorax for long hours and track the administration of exogenous surfactant as well as the treatment response of the patient. This could result in optimized timing of rescue doses of surfactant and prevention of alveolar collapse.

Recent efforts have assessed minimally invasive surfactant administration to provide surfactant without intubation and thus avoiding mechanical ventilation [16]. The results of such studies are promising. GASMAS technology may have a very important additional role to play in optimizing surfactant administration, mechanical ventilation techniques, endotracheal tube placement if required, and an additional tool to enhance extubating thresholds and strategies to prevent reintubation. Chronic Lung Dysfunction (CLD) usually evolves in preterm infants from their RDS. It is a consequence of the improved survival of new borns and the most relevant specific condition that adversely affects postnatal lung development. Risk factors for CLD are mechanical ventilation, fluid overload and hyperoxia [17]. Inflammation is an indication that CLD will develop. GASMAS technology is also capable of measuring inflated volume in the lung and can potentially quantify inflammatory changes within the lungs of preterm infants. This would present a huge step in the optimization of clinical care, since chest radiography, the gold standard for diagnosis, might not always correlate with the clinical severity in CLD [7]. Postnatal infections, including sepsis and pneumonia are also risk factors for CLD where lung injury is thought to be mediated by inflammation [18]. Further studies could determine if infection of lung tissue has a gaseous biological marker. If so, GASMAS technology could optimize antibiotics administration.

\section{Conclusion}

The last advances in laser, photodetector and software technology make it possible to design a bedside GASMAS device with compact source and detector probes, capable of measuring oxygen concentration and lung volume in real time. The probes can be placed in remittance geometry for different positions over the thorax, allowing the mapping of alveolar performance over the different lung lobes. A bedside clinical device combining imaging features from ultrasound and the quantification gas composition and inflated volume of the lung offered by GASMAS has the potential 
to achieve a thorough lung function assessment of new-borns and help in the diagnosis of underlying respiratory conditions, diminishing the need of X-ray imaging. GASMAS technology may have a very important role to play in optimizing respiratory support for preterm infants and potentially decrease the incidence of CLD.

\section{Acknowledgements}

The authors would like to thank Jacqueline Gunther, Katarzyna Komolibus and Eugene Dempsey for valuable discussions. The research leading to these results was funded by Science Foundation Ireland project no. SFI/15/RP/2828.

\section{Conflict of Interest}

The authors certify that they have no affiliations with or involvement in any organization or entity with any financial interest in the subject matter or materials discussed in this manuscript.

\section{Reference}

1. (2018) World Health Organization.

2. Sweet DG, Carnielli V, Greisen G, Hallman M, Ozek E, et al. (2017) European Consensus Guidelines on the Management of Respiratory Distress Syndrome-2016 Update. Neonatology 111(17): 107-125.

3. Joshi S, Kotecha S (2007) Lung Growth and Development. Early Human Development 83: 789-794.

4. Gallacher DJ, Hart K and Kotecha S (2016) Common respiratory conditions of the newborn. Breathe 12(1): 30-42.

5. Kamath BD, Macguire ER, McClure EM, Goldenberg RL, Jobe (2011) Neonatal Mortality from Respiratory Distress Syndrome: Lessons for Low-Resource Countries. Pediatrics 127(6): 1139-1146.

6. Dyer J (2019) Neonatal Respiratory Distress Syndrome: Tackling A Worldwide Problem. P\&T 44(1): 12-14.

7. Rossi U, Owens C (2005) The radiology of chronic lung disease in children. Arch Dis Child 90(6): 601-607.
8. Hahn AD, Higano NS, Walkup LL, Thomen RP, Cao X, et al. (2017) Pulmonary MRI of neonates in the intensive care unit using 3D ultrashort echo time and a small foot-print MRI system. J Magn Reson Imaging 45(2): 463-471.

9. Liang H, Liang X, Chen Z, Tan X, Yang H, et al. (2018) Ultrasound in neonatal lung disease. Quant Imaging Med Surg 8(5): 535-546.

10. Vergine M, Copetti R, Brusa G, Cattarossi L (2014) Lung Ultrasound Accuracy in Respiratory Distress Syndrome and Transient Tachypnea of the Newborn. Neonatology 106(2): 87-93.

11. Svanberg EK, Lundin P, Larsson M, Åkeson J, Svanberg K, et all. (2016) Diode laser spectroscopy for noninvasive monitoring of oxygen in the lungs of newborn infants. Pediatric Research 79(4): 621-628.

12. Rothman LS, Gordon IE, Babikov Y, Barbe A, Benner DC, et all. (2013) The HITRAN 2012 molecular spectroscopic database. JQSRT 130: 4-50.

13. Svanberg S (2013) Gas in scattering media absorption spectroscopy from basic studies to biomedical applications. Laser Photonics Rev 7(5): 779-796.

14. Lewander M, Bruzelius A, Svanberg S, Svanberg K, Fellman V (2011) Nonintrusive Gas Monitoring in Neonatal Lungs Using Diode Laser Spectroscopy: Feasibility Study. J Biomed Opt 16(12): 127002.

15. Persson L, Lewander M, Andersson M, Svanberg K, Svanberg S (2008) Simultaneous detection of molecular oxygen and water vapor in the tissue optical window using tunable diode laser spectroscopy. Applied Optics 47(12): 2028-2034.

16. Aguar M, Nuñez A, Cubells E, Cernada M, Dargaville PA, et al. (2014) Administration of Surfactant Using Less Invasive Techniques as a Part of a Non-Aggressive Paradigm Towards Preterm Infants. Early Hum Dev 90(2): S57-59.

17. Bhandari V (2010) Hyperoxia-derived lung damage in preterm infants. Semin Fetal Neonatal Med 15(4): 223-229.

18. Van Marter LJ, Dammann O, Allred EN, Leviton A, Pagano M, et al. (2002) Chorioamnionitis, Mechanical Ventilation, and Postnatal Sepsis as Modulators of Chronic Lung Disease in Preterm Infants. J Pediatr 140(2): 171-176. 